\title{
The resonant damping of fast magnetohydrodynamic oscillations in a system of two coronal slabs
}

\author{
Iñigo Arregui ${ }^{1}$, Jaume Terradas $^{1,2}$, Ramón Oliver ${ }^{1}$, and José Luis Ballester ${ }^{1}$
}

\begin{abstract}
Observations of transversal coronal loop oscillations very often show the excitation and damping of oscillations in groups of coronal loops rather than in individual and isolated structures. We present results on the oscillatory properties (periods, damping rates, and spatial distribution of perturbations) for resonantly damped oscillations in a system of two inhomogeneous coronal slabs and compare them to the properties found in single slab loop models. A system of two identical coronal loops is modeled, in Cartesian geometry, as being composed by two density enhancements. The linear magnetohydrodynamic (MHD) wave equations for oblique propagation of waves are solved and the damping of the different solutions, due to the transversal inhomogeneity of the density profile, is computed. The physics of the obtained results is analyzed by an examination of the perturbed physical variables. We find that, due to the interaction between the loops, the normal modes of oscillation present in a single slab split into symmetric and antisymmetric oscillations when a system of two identical slabs is considered. The frequencies of these solutions may differ from the single slab results when the distance between the loops is of the order of a few slab widths. Oblique propagation of waves weakens this interaction, since solutions become more confined to the edges of the slabs. The damping is strong for surface-like oscillations, while sausage body-like solutions are unaffected. For some solutions, and small slab separations, the damping in a system of two loops differs substantially from the damping of a single loop.
\end{abstract}

Subject headings: MHD — Sun: corona — Sun: magnetic fields — waves

\footnotetext{
${ }^{1}$ Departament de Física, Universitat de les Illes Balears, E-07122 Palma de Mallorca, Spain. Email: inigo.arregui@uib.es, jaume.terradas@uib.es, ramon.oliver@uib.es and dfsjlb0@uib.es

${ }^{2}$ Centrum voor Plasma Astrofysica, K.U. Leuven, Celestijnenlaan 200B, B-3001 Heverlee, Belgium
} 


\section{INTRODUCTION}

The solar corona, the outermost part of the solar atmosphere, is able to support a variety of MHD waves in its different magnetic and plasma configurations. In the last years, particular attention has been devoted to the phenomenon of transversal coronal loop oscillations, first observed by instruments on-board TRACE spacecraft (Aschwanden et al. 1999; Nakariakov et al. 1999; Aschwanden et al. 2002; Schrijver et al. 2002). These oscillations, triggered mainly by nearby flares or filament eruptions, consist of lateral displacements of the loops while their foot-points are fixed to the denser photosphere. The characteristic periods are of the order of minutes and oscillations are quickly damped in a few periods.

From a theoretical point of view, these oscillations have been interpreted by Nakariakov et al. (1999) as the fundamental fast MHD kink mode of a flux tube. This solution involves a global motion of the whole structure that displaces the axis of the tube. The identification of observed oscillations with theoretical MHD wave solutions is the key for coronal seismology, first suggested by Uchida (1970); Roberts et al. (1984), and has allowed preliminary estimations of the magnetic field strength, transport coefficients, and plasma- $\beta$ in coronal loops (Nakariakov et al. 1999; Nakariakov \& Ofman 2001; Aschwanden 2005). Some recent examples of the application of coronal seismology can be found in Nakariakov \& Ofman (2001); Goossens et al. (2002); Andries et al. (2005); Verwichte et al. (2006); Arregui et al. (2007a). As for the nature of the damping mechanism(s) there is little consensus yet and several mechanisms are currently under study, such as non-ideal effects, lateral wave leakage due to the curvature of the loops (Terradas et al. 2006), mechanisms based on the topology of the magnetic field lines (Schrijver \& Brown 2000), and resonant conversion of wave energy, due to the non-uniformity of the magnetic-plasma configuration (Hollweg \& Yang 1988; Ruderman \& Roberts 2002; Goossens et al. 2002, 2006). Foot-point leakage through the chromospheric density gradient (De Pontieu et al. 2001; Ofman 2002) has been invoked for the damping of Alfvén waves and could be ascribed to the damping of transverse oscillations, as well.

Observations of transversal coronal loop oscillations very often show the excitation and damping of motions in groups of coronal loops rather than in single, isolated structures (see for example the event recorded by TRACE on 2001 April 15, analyzed by Verwichte et al. 2004). However, most of the theoretical models used for their study are based on single loop models. The interaction between the motions of adjacent coronal loops may change the oscillatory properties (periods, damping times, and spatial distribution of perturbations) of the observed event. For this reason, it is important to fully understand the theoretical properties of MHD waves in multiple-loop structures. The interest of these studies is two-fold. On one hand, to see under which conditions these differences in the oscillatory properties 
are important and, on the other hand, if important, to make reliable predictions and determinations of unknown physical parameters in the corona. For example, when analyzing the 2001 April 15 event, Verwichte et al. (2004) report on damping times that are longer than expected from previous observations of transverse oscillations in isolated loops. The fact that the studied coronal loops belong to a group could be behind this result.

Several authors have previously investigated the properties of MHD waves in multistructures. Berton \& Heyvaerts (1987) studied the properties of the normal modes of oscillation of a periodic medium. Bogdan \& Fox (1991) and Keppens et al. (1994) considered the scattering and absorption of acoustic waves by bundles of magnetic flux tubes with sunspot properties. Murawski (1993) and Murawski \& Roberts (1994) studied, by means of numerical simulations, the propagation of fast waves in a system of two slabs unbounded in the longitudinal direction. The collective nature and properties of oscillations in multi-fibril Cartesian systems was analyzed by Díaz et al. (2005), in the context of prominence oscillations. More recently, Ofman (2005) performed numerical simulations for the damping of a bundle of four loops, Marcu et al. (2006) have extended the study by Berton \& Hevvaerts (1987) by including a steady motion in the equilibrium and Ogrodowczyk \& Murawski (2007) have performed numerical simulations of impulsively excited magneto-sonic waves in two parallel solar coronal slabs. Also recently, Luna et al. (2006) have explored the normal modes and the time-dependent evolution of fast kink MHD perturbations in a system of two coronal loops, modeled as plasma slabs. They find a splitting of modes into symmetric and antisymmetric kink-like oscillations. The interaction between two coronal loops produces a variation on the frequency and spatial structure of the eigenfunctions, that depend now on the distance between the loops.

In this paper, we present the oscillatory properties of fast MHD waves in a system of two Cartesian slabs when both oblique propagation of perturbations and transversal inhomogeneity of the medium are included. These two ingredients produce the resonant damping of fast waves. A particular study of the oscillatory properties of a similar physical system was recently presented by Arregui et al. (2007b), who considered two very close slabs to analyze the effect of the possibly unresolved internal structuring of coronal loops on their transverse damped oscillations. Here a more in-depth analysis is performed. The resonant damping of fast MHD oscillations in a single Cartesian slab has been described by Arregui et al. (2007c). In this paper, a second density enhancement is included and an analogous analysis is presented. This work, thus, constitutes an extension to Luna et al. (2006) to the case of oblique propagation and damping by resonant absorption and an extension to Arregui et al. (2007c) to a system of two interacting slabs. Although the model adopted in this work is an oversimplification, and is far from being an accurate representation of real coronal loop systems, this simplicity allows a detailed study of the problem. 
The layout of the paper is as follows. In $\S 2$, the equilibrium configuration, the linear MHD wave equations for coupled fast and Alfvén waves, and a description of the numerical method are presented. Then, $\S 3$ describes the results of our study. First, in $\S 3.1$, the normal mode properties of undamped fast waves for oblique propagation are described. Then, in $\S$ 3.2, the damping properties of the solutions and the physical interpretation of the results are discussed. Finally, in $\S$ 4, our conclusions are drawn.

\section{EQUILIBRIUM MODEL, LINEAR MHD WAVE EQUATIONS, AND NUMERICAL METHOD}

We model the equilibrium magnetic and plasma configuration of a system of two coronal loops by means of a one-dimensional model in Cartesian geometry. The magnetic field is straight and pointing in the $z$-direction, $\mathbf{B}=B \hat{\mathbf{e}}_{\mathrm{z}}$. For applications to the solar corona, it is a good approximation to consider that the magnetic pressure dominates over the gas pressure. This classic zero plasma- $\beta$ limit implies that the magnetic field is uniform and that the density, $\rho(x)$, or Alfvén speed, $v_{\mathrm{A}}(x)$, profiles can be chosen arbitrarily. For simplicity, we consider a system of two identical coronal loops. This system is then modeled by defining a particular equilibrium density profile in the $x$-direction (see Figure 1), with two density enhancements of half-width $a$ located at $\pm x_{0}$. The density in each of the slabs is uniform, $\rho_{i}$, and connected to the uniform coronal environment, with density $\rho_{e}$, by transitional nonuniform layers of thickness $l$. The explicit expression for the considered equilibrium density is (for $x \geq 0$ )

$$
\rho(x)= \begin{cases}\rho_{e} & \text { if } 0 \leq x \leq x_{0}-a-\frac{l}{2} \\ f_{1}(x) & \text { if } x_{0}-a-\frac{l}{2} \leq x \leq x_{0}-a+\frac{l}{2} \\ \rho_{i} & \text { if } x_{0}-a-\frac{l}{2} \leq x \leq x_{0}-a+\frac{l}{2} \\ f_{2}(x) & \text { if } x_{0}+a-\frac{l}{2} \leq x \leq x_{0}+a+\frac{l}{2} \\ \rho_{e} & \text { if } x \geq x_{0}+a+\frac{l}{2}\end{cases}
$$

with $\rho(-x)=\rho(x)$. The particular dependence of the density profiles at the non-uniform transitional layers has no qualitative importance on the obtained results. We have chosen them, following Ruderman \& Roberts (2002) and Van Doorsselaere et al. (2004), as

$$
f_{1}(x)=\frac{\rho_{i}}{2}\left[\left(1+\frac{\rho_{e}}{\rho_{i}}\right)-\left(1-\frac{\rho_{e}}{\rho_{i}}\right) \sin \frac{\pi\left[-x-\left(-x_{0}+a\right)\right]}{l}\right]
$$

and 


$$
f_{2}(x)=\frac{\rho_{i}}{2}\left[\left(1+\frac{\rho_{e}}{\rho_{i}}\right)-\left(1-\frac{\rho_{e}}{\rho_{i}}\right) \sin \frac{\pi\left[x-\left(x_{0}+a\right)\right]}{l}\right]
$$

This equilibrium configuration is the same as the one used by Arregui et al. (2007b) for the study of the influence of the internal density structuring of a coronal loop on the properties of their damped oscillations. Their analysis is based on an equilibrium model in which the two slabs are very close to each other and contains a comparison of the results to the ones obtained with similar single slab models. This equilibrium model is also an extension of the one considered by Luna et al. (2006), with the addition of non-uniform density layers at the edges of each slab.

In order to study small amplitude oscillations of the previous equilibrium, the linear resistive MHD equations, with constant magnetic diffusivity, $\eta$, are considered. As the equilibrium configuration only depends on the $x$-direction, a spatial and temporal dependence of the form $\exp ^{\imath\left(\omega t-k_{y} y-k_{z} z\right)}$ is assumed for all perturbed quantities, with $\omega=\omega_{R}+\imath \omega_{I}$ the complex frequency and $k_{y}$ and $k_{z}$ the perpendicular and parallel wavenumbers. For resonantly damped solutions, the real part of the frequency gives the period of the oscillation, $P=2 \pi / \omega_{R}$, while the imaginary part is related to the damping time, $\tau_{d}=1 / \omega_{I}$. The photospheric line-tying effect is then included by selecting the appropriate parallel wavenumber. This leads to the following set of ordinary differential equations for the two components of the velocity perturbation, $v_{x}$ and $v_{y}$, and the three components of the perturbed magnetic field, $b_{x}, b_{y}$, and $b_{z}$,

$$
\begin{aligned}
\omega v_{x} & =\frac{B}{\rho}\left(-k_{z} b_{1 \mathrm{x}}+\imath \frac{d b_{1 \mathrm{z}}}{d x}\right) \\
\omega v_{y} & =\frac{B}{\rho}\left(-k_{z} b_{1 \mathrm{y}}+k_{y} b_{1 \mathrm{z}}\right) \\
\omega \frac{b_{1 \mathrm{x}}}{B} & =-k_{z} v_{x}-\imath \frac{\eta}{B}\left[\frac{d^{2} b_{1 \mathrm{x}}}{d x^{2}}-\left(k_{y}^{2}+k_{z}^{2}\right) b_{1 \mathrm{x}}\right] \\
\omega \frac{b_{1 \mathrm{y}}}{B} & =-k_{z} v_{y}-\imath \frac{\eta}{B}\left[\frac{d^{2} b_{1 \mathrm{y}}}{d x^{2}}-\left(k_{y}^{2}+k_{z}^{2}\right) b_{1 \mathrm{y}}\right] \\
\omega \frac{b_{1 \mathrm{z}}}{B} & =\left(\imath \frac{d v_{x}}{d x}+k_{y} v_{y}\right)-\imath \frac{\eta}{B}\left[\frac{d^{2} b_{1 \mathrm{z}}}{d x^{2}}-\left(k_{y}^{2}+k_{z}^{2}\right) b_{1 \mathrm{z}}\right] .
\end{aligned}
$$

These equations, together with the appropriate boundary conditions, define an eigenvalue problem for fast and Alfvén normal modes. As the plasma- $\beta=0$, the slow mode is absent 
and there are no motions parallel to the equilibrium magnetic field, $v_{z}=0$. In this paper, solutions to these equations are obtained by performing a normal mode analysis. When only perpendicular propagation of perturbations is considered $\left(k_{y} \neq 0, l=0\right)$, the solutions represent undamped fast MHD waves with oblique propagation, in addition to Alfvén waves. If non-uniform transitional layers are included $(l \neq 0)$, the solutions are damped. In this case, solutions are in general difficult to obtain analytically. Numerical approximations are then obtained using PDE2D (Sewell 2005), a general-purpose partial differential equation solver. The code uses finite elements and allows the use of a non-uniformly distributed grid, which is needed in order to properly resolve the large gradients that arise in the vicinity of resonant layers. This code has been used successfully in previous studies that involved similar computations of the damping rate by, for example, Terradas et al. (2006); Arregui et al. (2007b, c). As for the boundary conditions, we impose the vanishing of the perturbed velocity far away from the two-slab system, hence $\mathbf{v} \rightarrow 0$ as $x \rightarrow \pm \infty$.

\section{ANALYSIS AND RESULTS}

Solutions to Equations (3)-(7) for the simplest case, when $k_{y}=0$ and $l=0$, have been obtained by Luna et al. (2006). Fast normal modes are then decoupled from Alfvén

modes. Luna et al. (2006) find that the system is able to support a variety of oscillatory modes. In particular, they concentrate on the splitting of the kink transversal oscillation of a single slab, giving rise to two solutions, symmetric and antisymmetric with respect to $x=0$, in which the two slabs oscillate in the kink mode in phase or anti-phase with the same frequency. The fundamental mode is symmetric and its frequency is constrained between two limiting values. In the limit of no separation between slabs, it is equal to the kink mode frequency of a single slab with double width. As the distance between the slabs is increased, the interaction weakens and, for large separations, the two slabs do not "feel" each other and the frequency of the whole system is the same as the kink mode frequency of a single slab. The range of separation between slabs for which the interaction is strong is mainly determined by the parallel wavenumber, $k_{z}$, which determines the confinement and drop-off rate of the eigenfunctions in the coronal medium. These authors also find that the antisymmetric mode is not present for all the values of the distance between the slabs and becomes leaky when this distance is smaller than a given value, that depends on the adopted value for the parallel wavenumber.

When compared to Luna et al. (2006), there are two new ingredients in the physical model under consideration in this paper. On one hand, the non-uniformity of the equilibrium density at the edges of the slabs. On the other hand, the inclusion of perpendicular 
propagation of perturbations. These two ingredients produce the resonant coupling of fast modes to Alfvén modes and, as a result, the global modes of the system are damped. The damping of oscillations in a system of coronal loops has been reported in observations analyzed by Verwichte et al. (2004) and is likely to differ from the theoretical values obtained with isolated coronal loop models.

In $\S$ 3.1, we first describe the normal mode properties of oscillations of our two-slab system when oblique propagation of perturbations is included. Then, in $\S 3.2$, the damping of oscillations by resonant absorption and the properties of the eigenfunctions are detailed.

\subsection{Normal Modes in a Two-Slab System with Oblique Propagation}

We first consider solutions to Equations (3)-(7) including oblique propagation of waves $\left(k_{y} \neq 0\right)$ and in the absence of non-uniform transitional layers $(l=0)$. Also, $\eta=0$ is set in these equations. As the equilibrium is piece-wise constant there is no resonant coupling to Alfvén waves and solutions represent undamped oscillations. These solutions can be obtained by solving an analytical dispersion relation. Arregui et al. (2007c) have shown, for a single Cartesian slab, that oblique propagation of waves affects the nature and properties of kink- and sausage-like solutions. The fundamental kink mode, which is body for values of $k_{y}$ below certain value, becomes surface beyond that particular value. There is a sausage surface wave, with its frequency always below the internal cut-off frequency, which only exists when $k_{y} \neq 0$. The phase speed of these two solutions goes asymptotically to the kink speed for quasi-perpendicular propagation $\left(k_{y} \gg k_{z}\right)$. On the other hand, the sausage body solution, which is leaky in the long wave limit $\left(k_{z} a \ll 1\right)$, becomes trapped when a non-zero $k_{y}$ is included. This solution is always above the internal cut-off frequency and, hence, keeps its body character in the limit of quasi-perpendicular propagation, due to the existence of the sausage surface solution below the internal cut-off frequency. All these features are well displayed in Figure 2 of Arregui et al. (2007c).

When a second density enhancement is included, the kink, sausage surface, and sausage body solutions, described by Arregui et al. (2007c), split into symmetric and antisymmetric solutions with respect to $x=0$, giving six solutions that we name symmetric and antisymmetric; kink (with the two slabs oscillating in the kink mode), sausage surface (with the two

slabs oscillating in the sausage surface mode), and sausage body (with both slabs oscillating in the sausage body mode).

When finding our solutions, we consider fixed values for the density contrast of the twoslab system, $\rho_{i} / \rho_{e}=10$, and for the longitudinal wavenumber, $k_{z} a=\pi / 50$. For the observed 
kink oscillations with a wavelength double the length of the loop, this corresponds to a ratio of length to width $L / 2 a=25$. These are typical values for observed coronal loops for which transverse oscillations have been reported. We have studied the dependency of the frequency of these solutions as a function of the position of the slabs, related to the distance between the loops, and as a function of the perpendicular wavenumber. Figure 2 displays the obtained results. The splitting of the solutions can clearly be seen for the six modes. For the kink symmetric and antisymmetric solutions, the range of distances for which the interaction is strong and the deviation from the kink mode frequency of a single slab significant, is large in the absence of perpendicular propagation $\left(k_{y}=0\right)$. Even for distances of the order of $100 a$, the two solutions do not converge to that frequency. When perpendicular propagation is included the single slab kink mode frequency is approached for much smaller distances between slabs, in such a way that only for distances of the order of a few times $a$ the symmetric and antisymmetric solutions have frequencies that differ significantly from the kink frequency of a single slab. Also, the increase of the perpendicular wavenumber allows the existence of the kink antisymmetric mode for smaller distances between slabs, in comparison to the $k_{y}=0$ case, when it becomes leaky below $x_{0} \simeq 26 a$. As for the sausage surface and sausage body, symmetric and antisymmetric solutions, a similar result is obtained. They are plotted only for values of $k_{y} \neq 0$ because the surface solutions only exist when $k_{y} \neq 0$ and the body solutions are leaky below a certain value of $k_{y}$. We can also appreciate the splitting of the single slab solutions and the dependency of the interaction between the slabs and, hence, the deviation from the single slab frequencies as a function of the slab separation and perpendicular wavenumber. It is interesting to note that the fundamental solution in the case of kink and sausage surface solutions is the symmetric one, while it is the antisymmetric one in the case of the sausage body solutions. The right panels of Figure 2 display the frequencies of the six solutions as a function of $k_{y}$ for a fixed value of the distance between the slabs. As we increase the value of the perpendicular wavenumber both symmetric and antisymmetric solutions converge to the same frequencies and approach those of the kink, sausage surface, and sausage body solutions of a single slab in the limit of quasi-perpendicular propagation.

We have computed the difference between the frequency of the symmetric and antisymmetric solutions of the two-slab system and the kink and sausage solutions of a single slab. For the kink solutions, and considering for example $x_{0}=2 a$, the frequency of the symmetric mode is $15 \%$ smaller than the kink mode frequency of a single slab, when $k_{y}=0$. This difference decreases to $3 \%$ for $k_{y} a=1$. When the two-slab system is oscillating in the antisymmetric mode, its frequency is $13 \%$ larger that the kink mode frequency for $k_{y} a=0.5$, while it is only $4 \%$ for $k_{y} a=1$. As for the sausage surface solutions of the two-slab system, a deviation from the sausage surface frequency of a single slab around $5 \%$ is found, in 
the case of the symmetric solution with $k_{y} a=0.3$ and $x_{0}=2 a$. Finally, for the sausage body solutions of the two-slab system with $x_{0}=2 a$, the maximum difference occurs for the antisymmetric solution with $k_{y} a=0.5$ and is around $10 \%$.

The results displayed in Figure 2 can easily be interpreted by analyzing the spatial distribution of the corresponding eigenfunctions. Figures 3, 4, and 5] show the transversal component of the perturbed velocity and the total pressure perturbation for the kink, sausage surface, and sausage body, symmetric and antisymmetric solutions, for different values of the perpendicular wavenumber. In Figure 3 the solutions for the kink solutions are plotted for three values of the perpendicular wave number. For $k_{y}=0$, the transversal velocity is maximum at the internal part of the slabs, while it decreases exponentially outside. The total pressure perturbation has an antisymmetric profile in each slab, with a zero value at the center. When oblique propagation is included, there is an improved confinement of the eigenfunctions with a sharper drop-off rate in the external medium, a behavior also found by Díaz et al. (2003), in the context of prominence fibril oscillations. Also, the character of the solutions inside the slabs change, and the two solutions become surface-like solutions, with a decreasing amplitude of $v_{x}$ inside the slabs as $k_{y}$ is increased. This improved confinement of the solutions weakens the interaction between the slabs and results in a frequency of the two-slab kink solution closer to that of the kink mode of a single slab. Regarding the sausage surface and body-like solutions (Figures 4 and (5), we can appreciate that they have a similar spatial distribution of eigenfunctions. The main difference has to be found in the total pressure perturbation profiles, that are maximum at the center of each slab, for the sausage body solutions, while they peak at the edges of the slabs for the surface-like counterparts. An increase in the perpendicular wavenumber also produces an improved confinement, in the external regions, for these four types of modes. This also helps explaining why the interaction between the slabs weakens for increasing perpendicular wavenumber. However, in contrast to the kink solutions, now the transversal velocity component inside the slabs is not affected by the increase in $k_{y}$ and the solutions keep their surface or body character. Finally, oblique propagation of waves affects the magnitude of the total pressure perturbation of the sausage surface, symmetric and antisymmetric solutions (Figure 4), but not very much that of the sausage body, symmetric and antisymmetric, modes inside the density enhancements (Figure 5]).

In summary, the inclusion of perpendicular propagation of perturbations changes significantly the oscillatory properties of fast MHD waves in our two-slab system. First, it allows the existence of two sausage surface solutions that are not present when $k_{y}=0$. Also, it allows the existence of trapped sausage body solutions for slab separations for which they are leaky when $k_{y}=0$. Second, it produces an improved confinement of the solutions that, for the six modes, are better confined to the slabs, with a sharper drop-off rate in 
the coronal medium. This produces changes in the frequencies of the six modes and varies significantly the range of slab separations for which the difference between the symmetric and antisymmetric frequencies and the single slab frequencies are important. In the case of the kink solutions, the increase of the perpendicular wavenumber also changes the character of the solutions inside the slabs, and the modes become surface-like. Therefore, for a fixed value of the parallel wavenumber, both the separation between the loops and the value of the perpendicular wavenumber determine the strength of the interaction between the slabs and the departure of the obtained frequencies from the results obtained with a single loop model.

\subsection{Resonant Damping of Oscillations}

Observations of transversal coronal loop oscillations show that motions are rapidly damped in only a few periods (Aschwanden et al. 1999; Nakariakov et al. 1999). Coronal loops forming an arcade also show this property, such as reported by Verwichte et al. (2004). Resonant absorption of wave energy has been proposed as a mechanism for the damping of loop oscillations by Hollweg \& Yang (1988), prior to observational evidence, provided by TRACE observations, of damped transversal loop oscillations. Goossens et al. (2002) provided an interpretation to the damping observed in TRACE loop oscillations based on this mechanism. Although there is still no consensus on what causes the rapid damping of loop oscillations, several authors have presented comparisons between theory and observations that point out that resonant damping is a viable mechanism (see Goossens et al. 2002; Aschwanden et al. 2003; Arregui et al. 2007a, for example). This damping mechanism relies on the coupling of global fast motions and localized Alfvénic motions due to the non-uniformity of the medium in the transversal direction.

To study the damping of the normal modes described in $\S 3.1$, we now include nonuniform transitional layers that connect the internal and external densities at the edges of each slab $(l \neq 0)$. For the normal mode analysis of Equations (3)-(7), a small but finite value of the resistivity has to be provided when computing the damping of oscillations. The magnetic Reynolds number in the solar corona is believed to be of the order of $10^{14}$, hence the resistive diffusion time is very large. Such large values of $R_{m}$ are difficult to handle in any numerical scheme. On the other hand, as shown by Poedts \& Kerner (1991), the damping by resonant absorption becomes independent of resistivity, beyond a given value of $R_{m}$. In our numerical computations, resistivity has been chosen to be small enough for the imaginary part of the frequency to be independent of resistivity, so that we can be confident that solutions that correspond to the solar coronal large Reynolds numbers are obtained. 
This condition has been checked to a high accuracy and for the solutions presented in this paper a value for the magnetic Reynolds number $R_{m}=v_{A i} a / \eta$ (with $v_{A i}$ the internal Alfvén speed) in between $10^{6}$ and $10^{8}$ has been sufficient.

We now consider the six different solutions presented in $\S 3.1$ and compute their damping due to the resonant coupling to Alfvénic motions. The same parameter values for the equilibrium configurations are considered, but now non-uniform transitional layers of thickness $l / a=0.3$ have been included. The results of our computations are displayed in Figure 6 . The left panels of this figure show the imaginary part of the frequency, for the six collective solutions under consideration, as a function of the position of the centers of the slabs. As can be seen, the splitting of solutions into symmetric and antisymmetric modes produces these modes to have a damping different from that of the corresponding single slab solutions. These differences go to zero in the limit of large separation between the slabs. As for the kink solutions, the antisymmetric mode has a stronger damping than its symmetric counterpart. This is also true for the sausage-like solutions. The most significant differences on the damping of the kink symmetric solution are produced for small distances between the slabs. However, the difference of the symmetric mode damping with respect to the kink mode damping of a single slab is below $10 \%$. The kink antisymmetric solution damping, on the other hand, is more than double that of the kink mode of a single slab. Also significant are the differences between the sausage surface symmetric and antisymmetric damping and the corresponding sausage surface mode damping of a single slab. The imaginary parts for these two modes are $70 \%$ smaller and larger, respectively, than the value for the single slab mode. The right panels of Figure 6 display the damping per period of the six solutions as a function of the perpendicular wavenumber, for a fixed value of the distance between the slabs. As we increase the value of perpendicular wavenumber the damping for both symmetric and antisymmetric solutions approaches the damping of the kink, sausage surface, and sausage body solutions of a single slab, in the limit of quasi-perpendicular propagation. Apart from the difference in the damping of these solutions with respect to those of the single slab and the dependence of the damping with perpendicular wavenumber, the most important result is that surface-like solutions, kink and sausage alike, are clearly strongly damped, within a few periods, for large values of $k_{y}$, while sausage body solutions are unaffected by resonant couplings (see Figure 6, bottom row). The reason for the absence of resonant damping in the case of the two sausage body solutions is that their frequencies lie outside the Alfvén continua produced by the presence of the four non-uniform layers. This leads to damping times, for these sausage solutions, that are of the order of the used Reynolds number and several orders of magnitude larger that the periods of oscillation.

In order to better understand the results displayed in Figure [6 and to get physical insight into the reason(s) for the different damping rates of the different solutions, we have 
analyzed the spatial distribution of the eigenfunctions for the six kind of solutions. Figures 7 . 8, and 9 display example eigenfunctions for the six types of solutions, for a particular set of parameters. The spatial distribution of eigenfunctions is rather similar to the ones shown in Figures 3, 4, and 5, for undamped solutions, but now peaks at the non-uniform transitional layers are clearly visible in the perturbed transverse velocity of the kink and sausage surface modes, while they are absent in the case of the two sausage body modes. These peaks are an indication of resonant coupling to Alfvén waves at the non-uniform layers. Aside from these peaks, the spatial distribution of the magnetic pressure perturbation has extrema at the four resonant layers in the case of the kink and the sausage surface modes, while it is maximum at the centers of the slabs in the case of the sausage body modes. The total pressure perturbation has no extrema at the resonant layers in the case of the sausage body solutions. The behavior of the eigenfunctions at the resonant layers and the fundamental conservation laws that govern the resonant couplings were studied by Sakurai et al. (1991) in ideal MHD and by Goossens et al. (1995) in dissipative MHD. These authors find that the derivative of the total pressure perturbation is zero at the resonant positions. This behavior is retrieved in the pressure perturbation for our damped solutions, but not in the two solutions for which resonant couplings are absent.

\section{SUMMARY AND CONCLUSIONS}

We have presented numerical results from a normal mode analysis for resonantly damped fast MHD oscillations in a system of two coronal loops. For simplicity, the adopted equilibrium model consists of two identical density enhancements in Cartesian geometry. The linearized resistive MHD wave equations are considered and solutions for obliquely propagating waves are obtained. The inclusion of oblique propagation together with non-uniform transitional layers that connect the internal and external densities produce the resonant damping of oscillations.

The considered equilibrium supports a variety of different wave solutions. The normal modes of oscillation in a two-slab system are, in general, different to those of a single and isolated slab, due to the interaction between the slabs. The well-known fast kink and sausage normal modes, present in a single slab configuration, split into symmetric and antisymmetric solutions. We have concentrated our analysis on the six solutions coming from the splitting of the kink and sausage (body and surface) modes in a single slab with oblique propagation, described by Arregui et al. (2007c). The frequencies of these six symmetric and antisymmetric solutions are in general different from the single slab solutions. They depend upon

the distance between the slabs (as shown by Luna et al. 2006, for the kink symmetric and 
antisymmetric solutions), but also on the magnitude of the perpendicular wavenumber. The inclusion of oblique propagation of perturbations has important effects on the dynamics of the system. First, it introduces two new surface-like solutions, with sausage parity, not present in the case of parallel propagation. Second, it decreases the effect of the interaction between the slabs, since solutions are more confined to the neighborhood of the density enhancements. Both the distance between slabs and perpendicular propagation determine the difference in frequency between the single slab model solutions and the modes described in this paper. These differences are of the order of $15 \%$ at most and should, therefore, be taken into account in the modeling of these events. They are, however, well below the observational uncertainties for the measured periods in transversal coronal loop oscillations, which are of the order of $40 \%$ (Aschwanden et al. 2002).

Next, we have studied the damping of the different solutions due to resonant coupling to Alfvénic motions. The period and damping rate of the six solutions have been computed and the spatial structure of eigenfunctions analyzed in order to find some physical insight into the obtained results. We find that surface-like solutions, both kink and sausage, are strongly damped. On the other hand, the two sausage body solutions are unaffected by resonant absorption. This is due to the absence of resonance due to the fact that the frequencies of these solutions are outside the corresponding Alfvén continua. A comparison with the single slab model results reveals that differences in damping are important for small values of the distance between the slabs. The most significant differences arise for the kink antisymmetric solution and the two sausage surface solutions, with differences in the imaginary part of the frequency of the order of $125 \%$ and $70 \%$ with respect to the damping of the corresponding single slab modes. These differences are of the order or larger than the observational uncertainties for the measured damping times in transversal coronal loop oscillations, which are around 60\% (Aschwanden et al. 2002).

In this work a magnetic two-slab system has been used to model a two-loop structure. Some of the results found in slab geometry cannot be translated to cylindrical coronal loop systems, while some others give a good approximation to the phase speeds in cylinders. For instance, the sausage surface solutions described in this paper are not present in a system of two cylinders. Also, the sausage body solutions would correspond to perturbations in each cylinder with $m=0$, thus they cannot be resonantly damped solutions. On the other hand, slabs are known to be poor wave-guides in comparison with cylinders. However, our results indicate that the inclusion of oblique propagation produces a sharper drop-off rate of the eigenfunctions in the external medium, resulting in a confinement similar to the case of cylinders and a good approximation of the phase-speed of the kink mode in a slender flux tube. The normal modes of oscillation in a system of two homogeneous cylinders have been computed recently by Luna et al. (2007). These authors find four solutions that correspond 
to transversal oscillations, symmetric and antisymmetric, in the plane containing the two cylinders. The frequency of these solutions is seen also to differ from the frequency of the kink mode for a single cylinder for small distances between the cylinders. A natural extension to the study presented in this paper and to the results described by Luna et al. (2007) is the analysis of the damping in a similar two-cylinder configuration.

The authors acknowledge the Spanish Ministerio de Educación y Ciencia for the funding

provided under project AYA2006-07637 and the Conselleria d'Economia, Hisenda i Innovació of the Government of the Balearic Islands for the funding provided under grants PRIB-200410145 and PCTIB2005GC3-03 (Grups Competitius). J. Terradas acknowledges the Spanish Ministerio de Educación y Ciencia for the funding provided under a Juan de la Cierva fellowship.

\section{REFERENCES}

Andries, J., Arregui, I., \& Goossens, M. 2005, ApJ, 624, L57

Arregui, I., Andries, J., Van Doorsselaere, T., Goossens, M., \& Poedts, S. 2007a, A\&A, 463, 333

Arregui, I., Terradas, J., Oliver, R., \& Ballester, J. L. 2007b, A\&A, 466, 1145

-. 2007c, Sol. Phys., in press

Aschwanden, M. J. 2005, Physics of the Solar Corona. An Introduction with Problems and Solutions (2nd edition) (Springer-Praxis)

Aschwanden, M. J., De Pontieu, B., Schrijver, C. J., \& Title, A. M. 2002, Sol. Phys., 206, 99

Aschwanden, M. J., Fletcher, L., Schrijver, C. J., \& Alexander, D. 1999, ApJ, 520, 880

Aschwanden, M. J., Nightingale, R. W., Andries, J., Goossens, M., \& Van Doorsselaere, T. 2003, ApJ, 598, 1375

Berton, R. \& Heyvaerts, J. 1987, Sol. Phys., 109, 201

Bogdan, T. J. \& Fox, D. C. 1991, ApJ, 379, 758

De Pontieu, B., Martens, P. C. H., \& Hudson, H. S. 2001, ApJ, 558, 859 
Díaz, A. J., Oliver, R., \& Ballester, J. L. 2003, A\&A, 402, 781

-. 2005, A\&A, 440, 1167

Goossens, M., Andries, J., \& Arregui, I. 2006, Royal Society of London Philosophical Transactions Series A, 364, 433

Goossens, M., Andries, J., \& Aschwanden, M. J. 2002, A\&A, 394, L39

Goossens, M., Ruderman, M. S., \& Hollweg, J. V. 1995, Sol. Phys., 157, 75

Hollweg, J. V. \& Yang, G. 1988, J. Geophys. Res., 93, 5423

Keppens, R., Bogdan, T. J., \& Goossens, M. 1994, ApJ, 436, 372

Luna, M., Terradas, J., Oliver, R., \& Ballester, J. L. 2006, A\&A, 457, 1071

-. 2007, ApJ, submitted

Marcu, A., Ballai, I., \& Pintér, B. 2006, A\&A, 449, 1193

Murawski, K. 1993, Acta Astronomica, 43, 161

Murawski, K. \& Roberts, B. 1994, Sol. Phys., 151, 305

Nakariakov, V. M. \& Ofman, L. 2001, A\&A, 372, L53

Nakariakov, V. M., Ofman, L., DeLuca, E. E., Roberts, B., \& Davila, J. M. 1999, Science, 285,862

Ofman, L. 2002, ApJ, 568, L135

—. 2005, Advances in Space Research, 36, 1572

Ogrodowczyk, R. \& Murawski, K. 2007, A\&A, 461, 1133

Poedts, S. \& Kerner, W. 1991, Phys. Rev. Lett., 66, 2871

Roberts, B., Edwin, P. M., \& Benz, A. O. 1984, ApJ, 279, 857

Ruderman, M. S. \& Roberts, B. 2002, ApJ, 577, 475

Sakurai, T., Goossens, M., \& Hollweg, J. V. 1991, Sol. Phys., 133, 227

Schrijver, C. J., Aschwanden, M. J., \& Title, A. M. 2002, Sol. Phys., 206, 69

Schrijver, C. J. \& Brown, D. S. 2000, ApJ, 537, L69 
Sewell, G. 2005, The Numerical Solution of Ordinary and Partial Differential Equations (Wiley-Interscience)

Terradas, J., Oliver, R., \& Ballester, J. L. 2006, ApJ, 650, L91

Uchida, Y. 1970, PASJ, 22, 341

Van Doorsselaere, T., Andries, J., Poedts, S., \& Goossens, M. 2004, ApJ, 606, 1223

Verwichte, E., Foullon, C., \& Nakariakov, V. M. 2006, A\&A, 452, 615

Verwichte, E., Nakariakov, V. M., Ofman, L., \& Deluca, E. E. 2004, Sol. Phys., 223, 77 


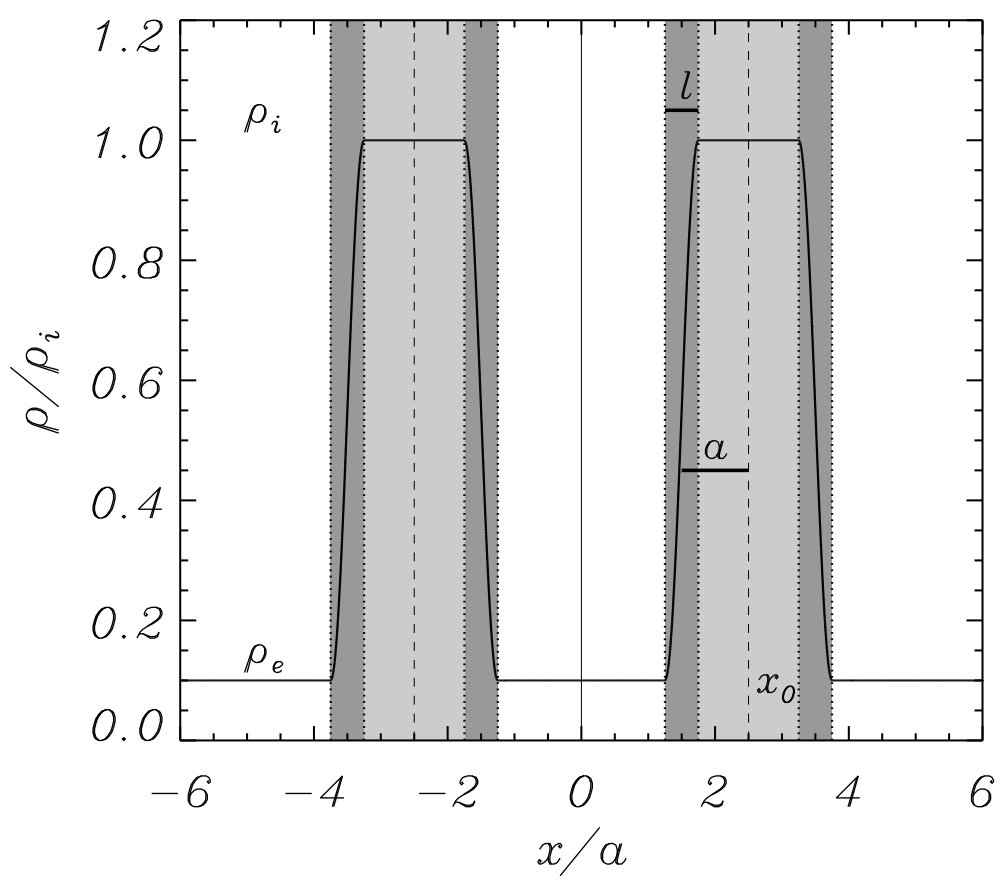

Fig. 1.- Schematic representation of the two density enhancements (light-shaded regions) of half-width $a$, centered at $\pm x_{0}$ representing a system of two coronal slabs in the direction transverse to the equilibrium magnetic field. These enhancements with internal density $\rho_{i}$ connect to the external medium, with density $\rho_{e}$, by transitional non-uniform layers (shaded regions) of thickness $l$. 

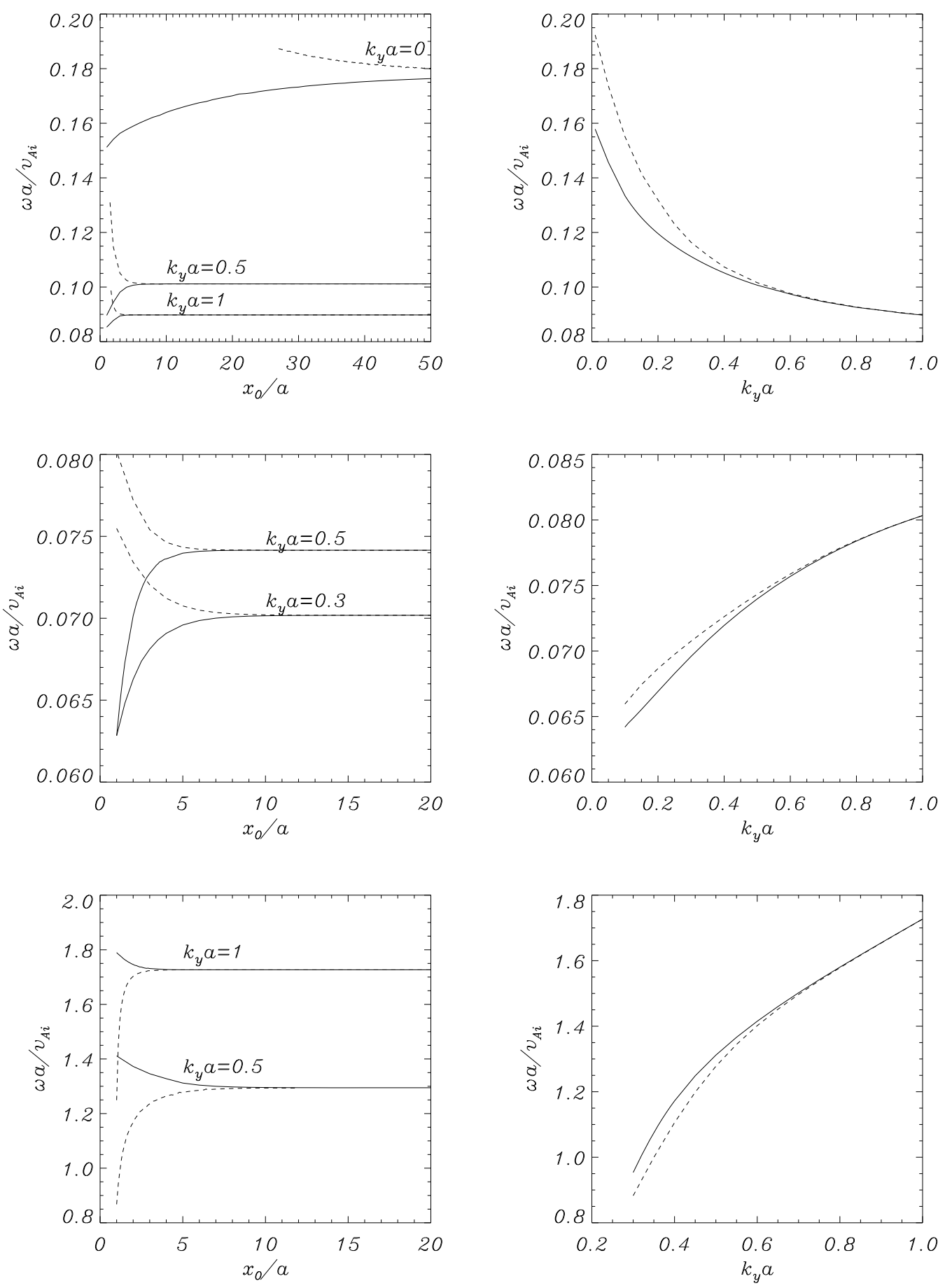

Fig. 2.- Left, Frequency as a function of the position of the slabs centers for different values of the perpendicular wavenumber for the kink solutions (top), the sausage surface solutions (middle), and the sausage body solutions (bottom). Right, Frequency as a function of the perpendicular wavenumber for the same six types of solution, for a fixed distance between the slabs, $x_{0}=5 a$. In all the figures the solid lines correspond to the symmetric solution and the dashed lines to the antisymmetric solution. 

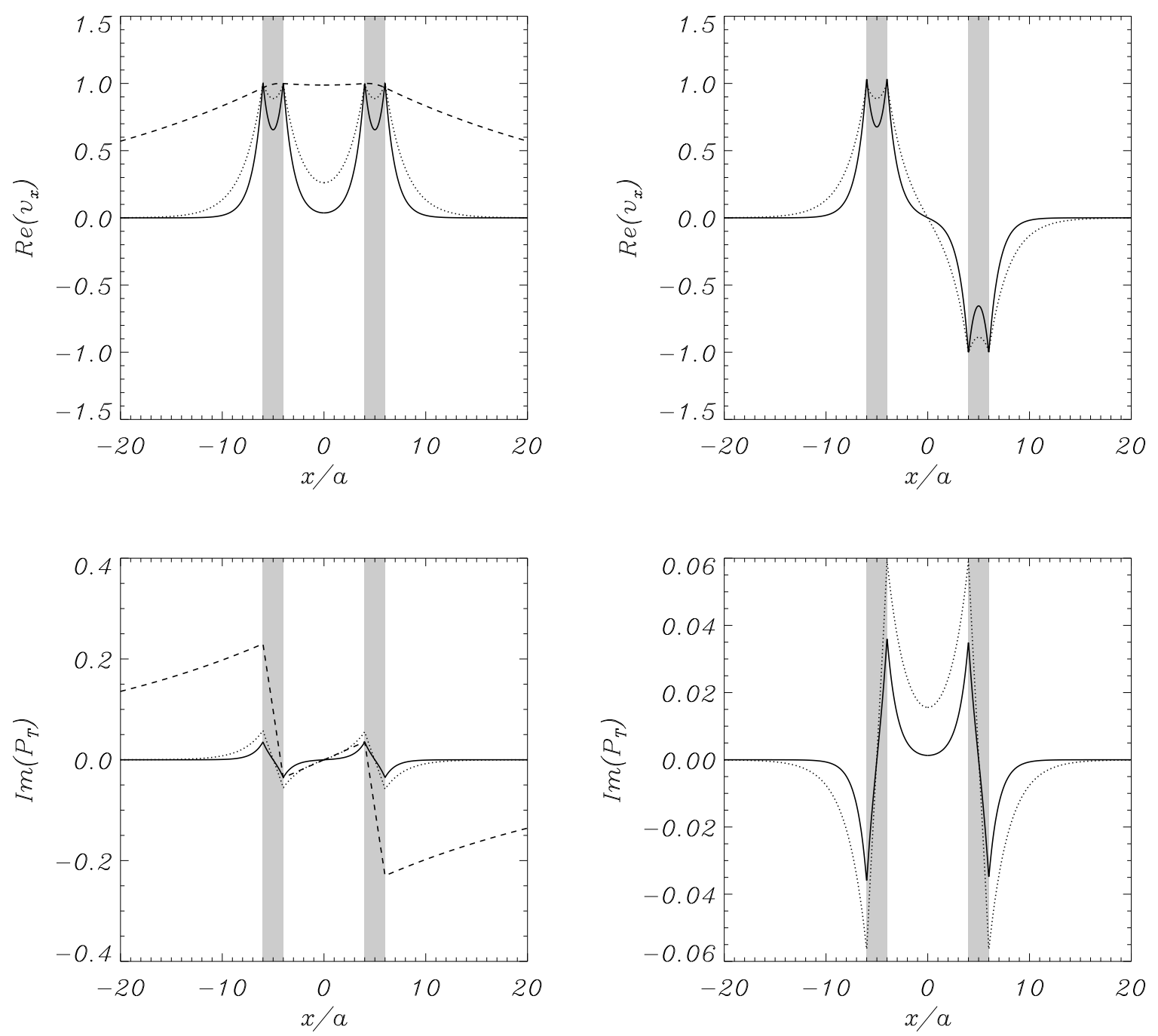

Fig. 3. - Real part of the transversal velocity component, $v_{x}$, and imaginary part of the perturbed total pressure, $P_{T}$, for the fundamental symmetric (left) and antisymmetric (right) kink solutions, for several values of the perpendicular wave number: $k_{y} a=0$ (dashed lines), $k_{y} a=0.5$ (dotted lines), and $k_{y} a=1$ (solid lines). Note that the character of the eigenfunctions for the symmetric mode changes from body-like to surface-like solution as $k_{y}$ is increased. In all figures the light-shaded regions represent the density enhancements. These solutions have been obtained with a uniform computational grid with $N_{x}=10000$ points in the range $-80 \leq x / a \leq 80$ (except for the kink solutions with $k_{y}=0$ for which a range $-200 \leq x / a \leq 200$ has been used). 

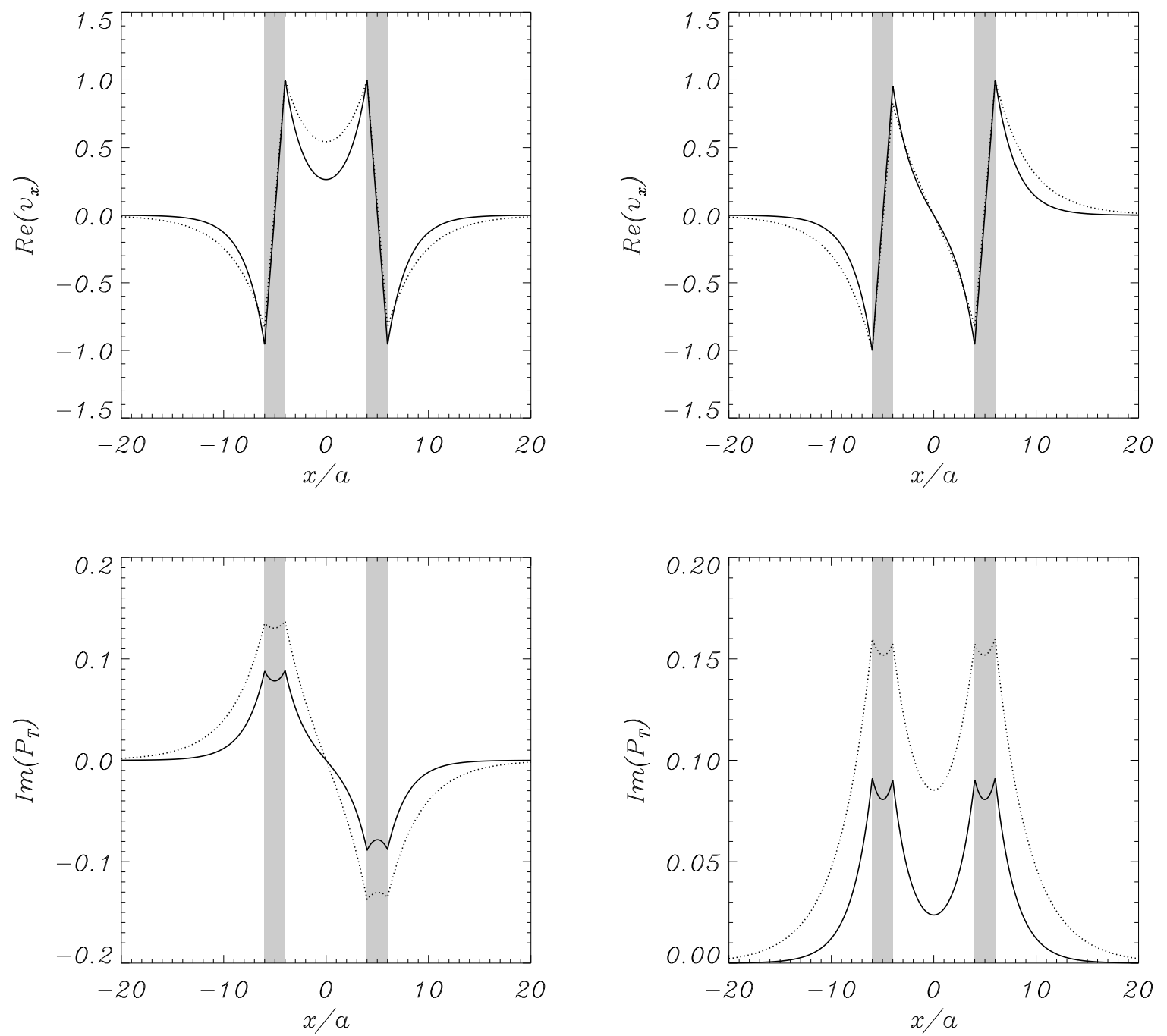

Fig. 4.- Same as Figure 3 for the sausage surface, symmetric (left) and antisymmetric (right) solutions and two values of the perpendicular wavenumber: $k_{y} a=0.3$ (dotted lines) and $k_{y} a=0.5$ (solid lines). These solutions are surface-like for all values of the perpendicular wavenumber. 

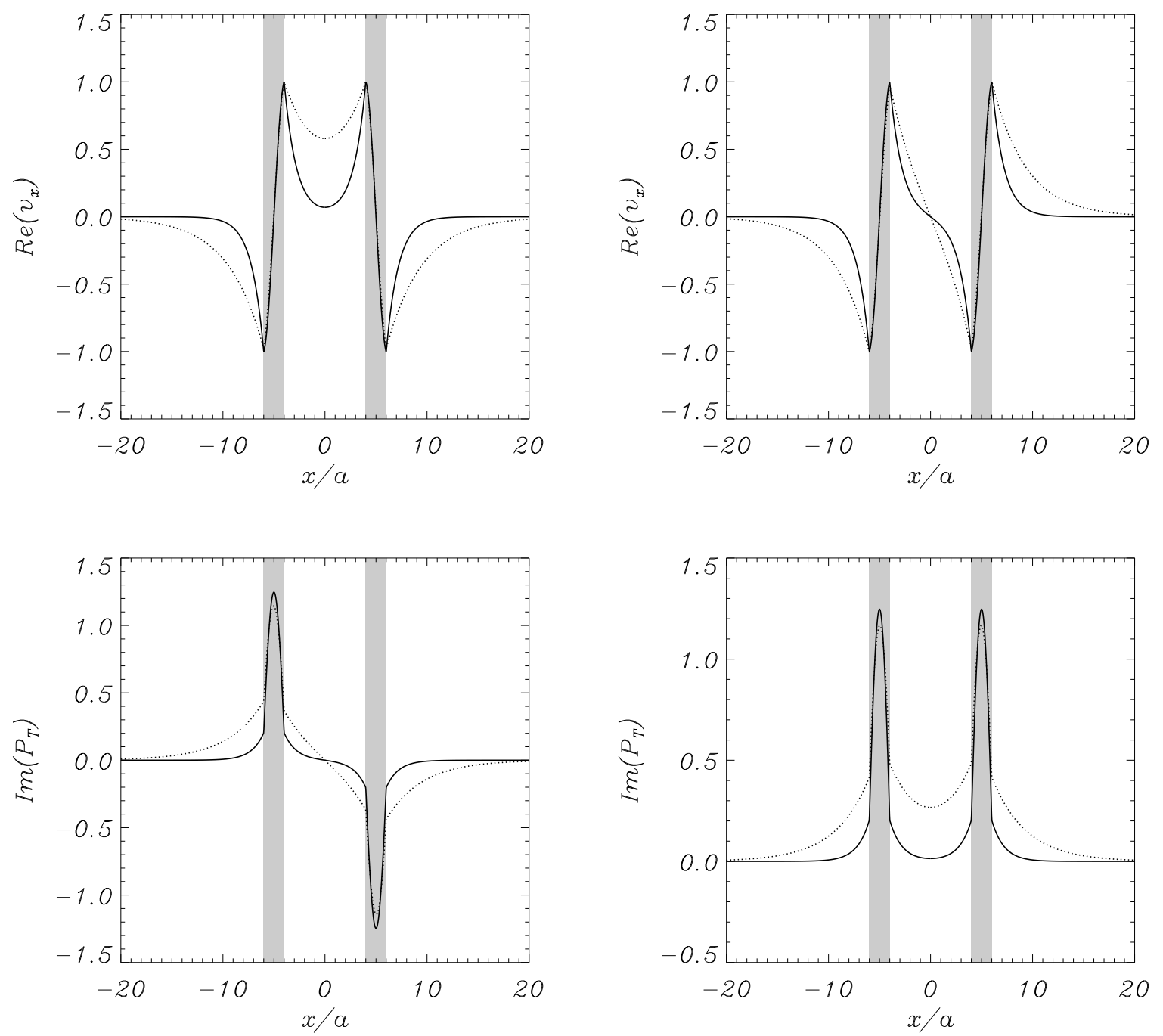

Fig. 5.- Same as Figures 3 and 4 for the sausage body, symmetric (left) and antisymmetric (right) solutions and two values of the perpendicular wavenumber: $k_{y} a=0.5$ (dotted lines) and $k_{y} a=1$ (solid lines). Note that for these solutions $v_{x}$ is very similar to the ones depicted in Figure 4, while the pressure perturbation in and around the slabs differ substantially. These solutions are body-like for all values of the perpendicular wavenumber. 

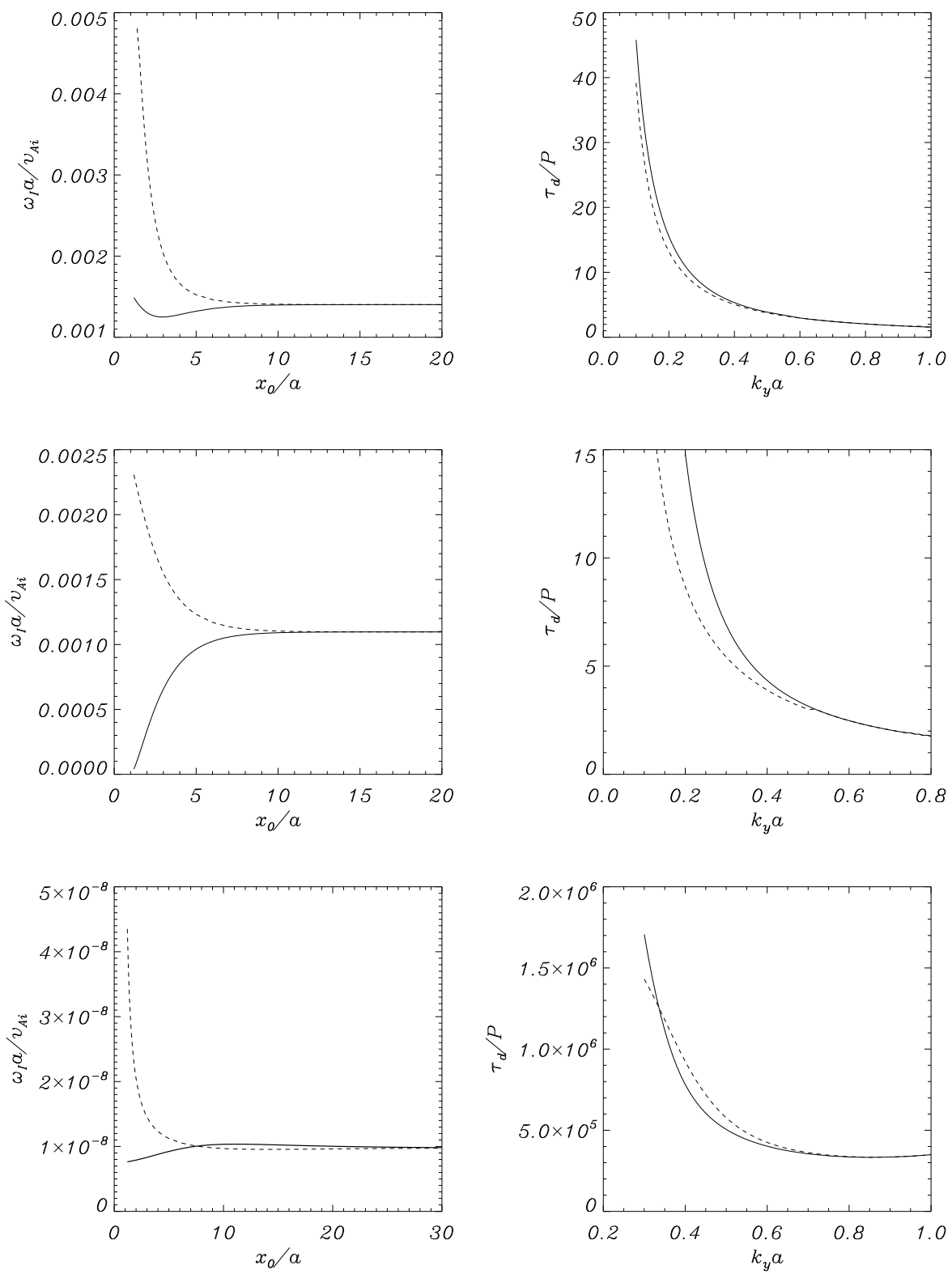

Fig. 6. - Left, Imaginary part of the frequency as a function of the position of the slabs centers for the kink solutions (top), the sausage surface solutions (middle), and the sausage body solutions (bottom) for $k_{y} a=0.3$ and $l / a=0.3$. Right, Damping time over period as a function of the perpendicular wavenumber for the same six types of solution, for the same parameter values and a fixed distance between the slabs, $x_{0}=5 a$. In all the figures the solid lines correspond to the symmetric solution and the dashed lines to the antisymmetric solution. A value for the magnetic Reynolds number $R_{m}=v_{A i} a / \eta=10^{7}$ has been used in a non-uniform grid with $N_{x}=14000$ points (2 000 in each of the four non-uniform transitional layers) in the range $-80 \leq x / a \leq 80$. 

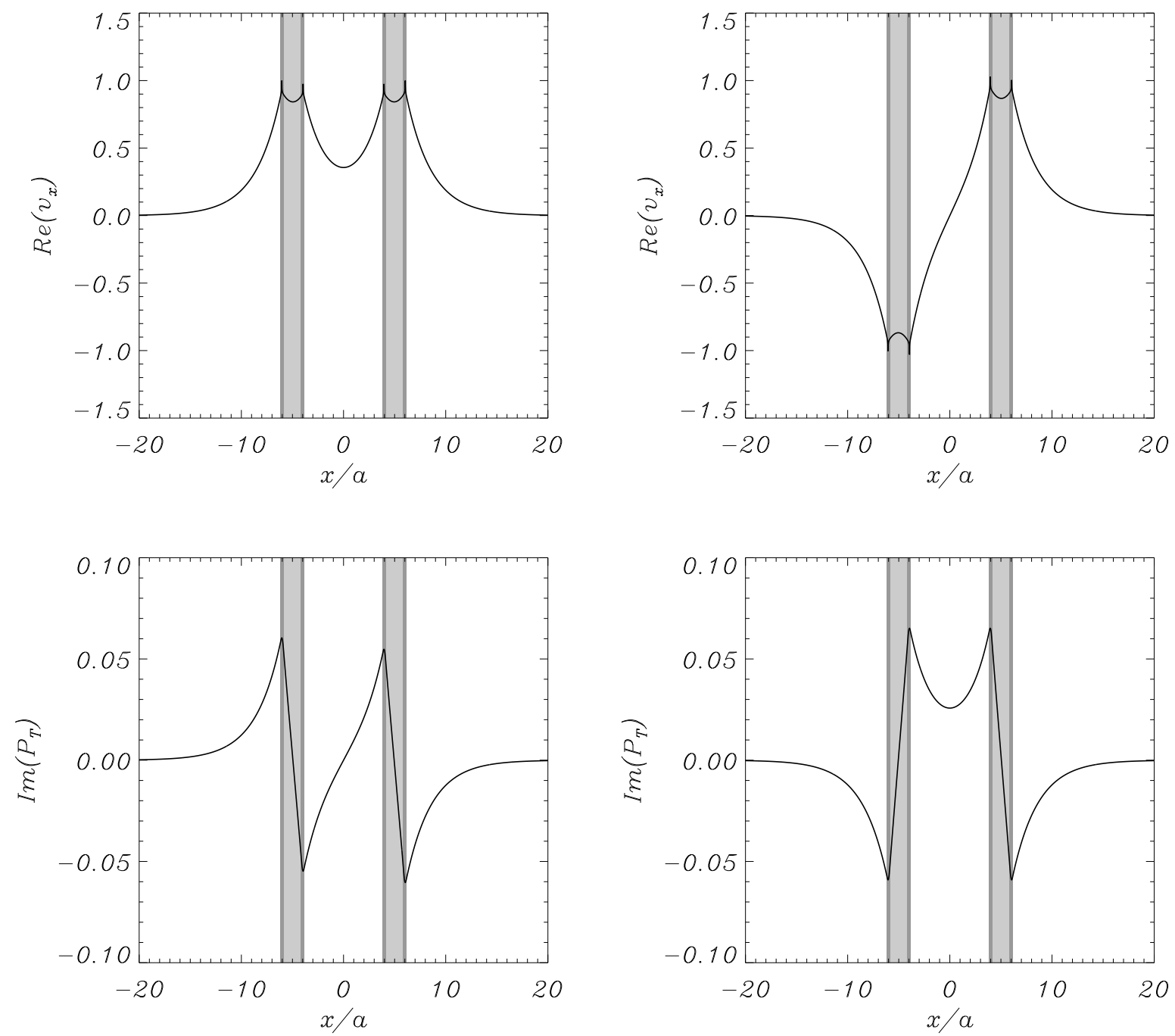

Fig. 7.- Real part of the transversal velocity component, $v_{x}$, and imaginary part of the perturbed total pressure, $P_{T}$, for the fundamental symmetric (left) and antisymmetric (right) kink solutions, for $k_{y} a=0.3$ and non-uniform transitional layers with thickness $l / a=0.3$. A value for the magnetic Reynolds number $R_{m}=v_{A i} a / \eta=10^{7}$ has been used in a non-uniform grid with $N_{x}=14000$ points ( 2000 on each of the four non-uniform transitional layers) in the range $-80 \leq x / a \leq 80$. In all figures the light-shaded regions represent the density enhancements and the shaded regions the non-uniform layers. 

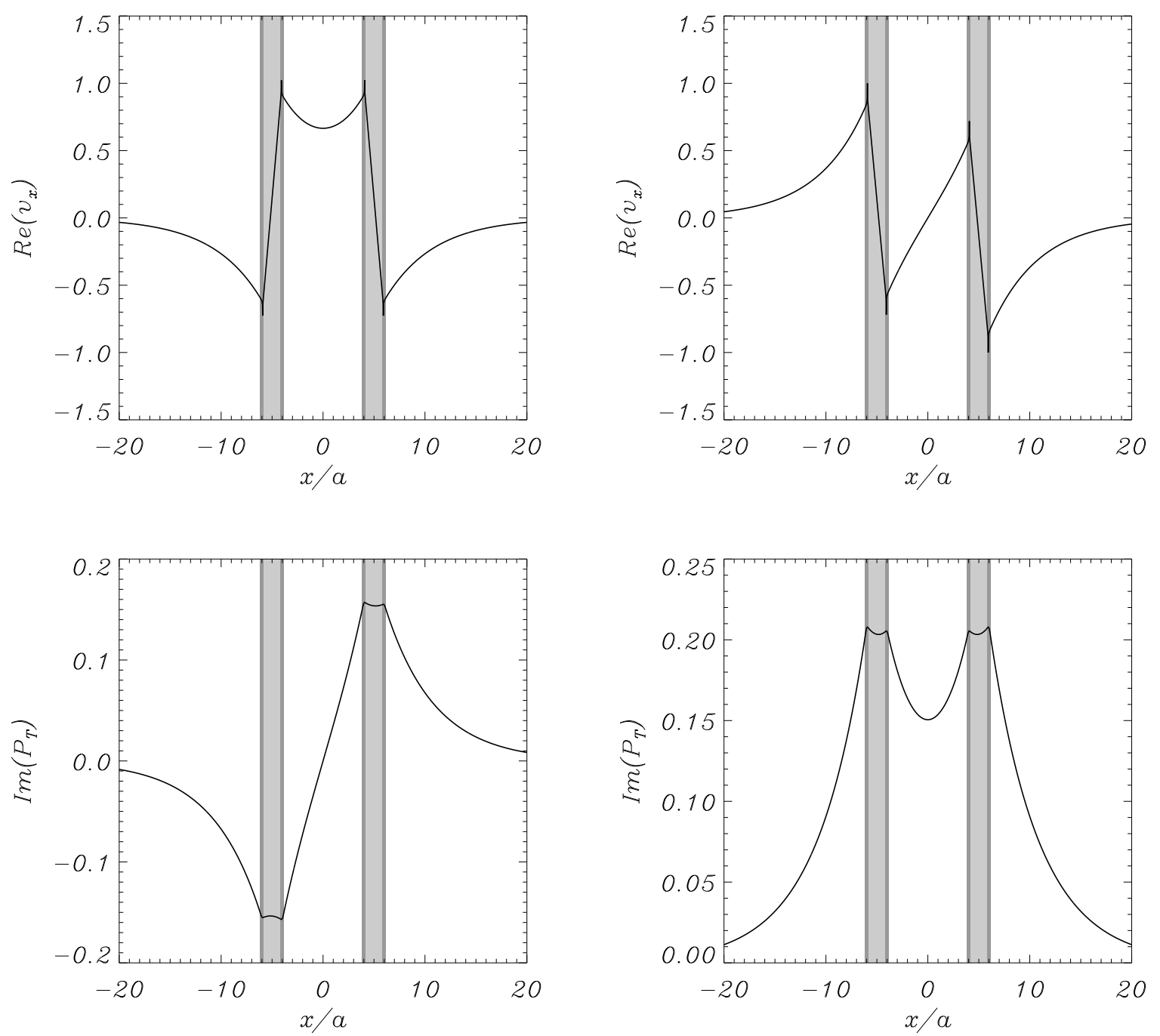

Fig. 8.- Same as Figure 7 for the fundamental symmetric (left) and antisymmetric (right) sausage surface solutions. 

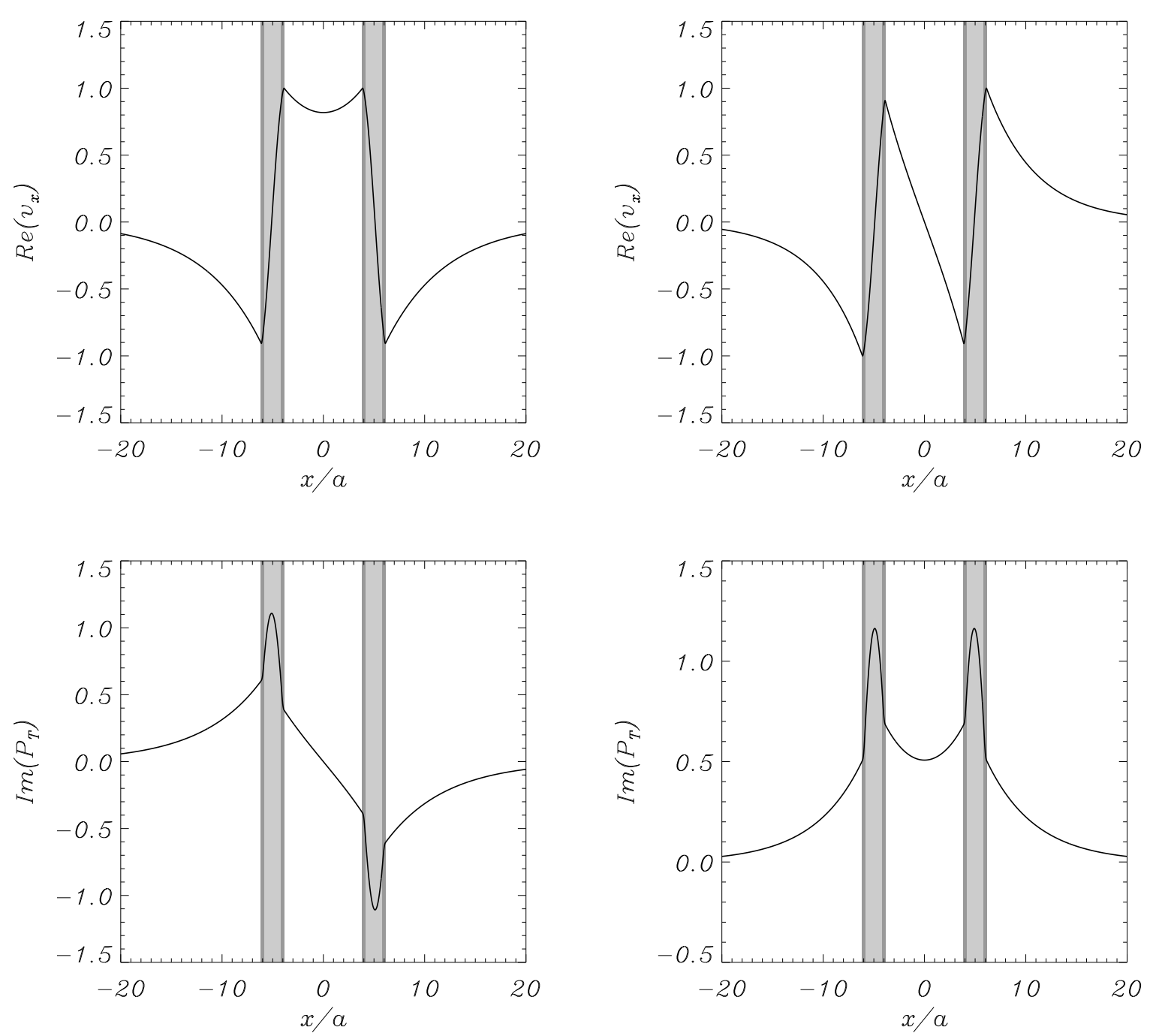

Fig. 9.- Same as Figures 7 and 8 for the fundamental symmetric (left) and antisymmetric (right) sausage body solutions. 